\title{
Die Geschichte der verschiedenen Rosengärten im Frankfurter Palmengarten
}

\author{
Beate TAudte-Repp
}

\begin{abstract}
The presentation of roses has a long tradition in the Palmengarten, originated in 1868. The abundant collections of plants from the Biebricher Wintergärten, held by Adolph, Duke of Nassau, which gave rise to the new garden in Frankfurt, already comprised about 2000 rosebushes. After a provisional rose arrangement in 1881, the first genuine rosarium was established in 1886. Further compounds and locations followed. In 1986, the Rose Garden has been assigned its current location (across from the entrance showhouse in Siesmayerstraße). A comprehensive restoration and replacement planting was accomplished in 2007. The following article is the long version of a contribution the author published in an anthology about rose gardens in Frankfurt and the surrounding Rhein-Main region (KüBLER 2011).
\end{abstract}

\section{Zusammenfassung}

Die Präsentation von Rosen hat im 1868 begründeten Palmengarten eine lange Tradition. Schon die reichen Pflanzensammlungen aus den Biebricher Wintergärten des Herzogs Adolph von Nassau, die seinerzeit den Grundstock für die neue Frankfurter Gartenschöpfung bildeten, enthielten 2000 Rosenstöcke. Nach einer provisorischen Rosenanlage von 1881 wurde das erste veritable Rosarium 1886 angelegt. Weitere Anlagen und Standorte folgten. 1986 erhielt der Rosengarten seinen heutigen Platz gegenüber dem Eingangsschauhaus an der Siesmayerstraße. 2007 wurde die Anlage umfassend saniert und neu bepflanzt. Der folgende Artikel ist die Langfassung eines Beitrages, den die Autorin in einem Sammelband über Rosengärten in Frankfurt und Rhein-Main (KüBLER 2011) veröffentlicht hat.

\section{Einführung: Rosen im Palmengarten}

Kein Garten ohne Rosen! Das gilt umso mehr für einen Garten, der mit seiner über 150 Jahre alten Tradition, seinen reichen botanischen Sammlungen sowie einem regen Ausstellungsund Veranstaltungsprogramm nicht nur in ganz Deutschland eine einmalige Einrichtung ist. Seit jeher ein Park zur Erholung, ein Ort der Bildung und Kultur ebenso wie ein botanischer Schaugarten, bildet der Frankfurter Palmengarten, der seit 1.1.2012 mit dem benachbarten Botanischen Garten vereinigt ist, dem Grüneburgpark und dem Parkareal des UniversitätsCampus am Poelzig-Bau eine stattliche grüne Schneise. Diese verläuft mitten durch das Westend der Main-Metropole. Etwa eine gute Viertelstunde Fußweg ist die Innenstadt entfernt. Deren Hochhaus-Skyline, die vielerorts im „Hortus Palmarum“ über den Baumkronen aufragt, bildet einen reizvollen architektonischen Widerpart zur üppigen Pflanzenwelt und auch zu den Rosen.

Seit ihrer Gründung kultiviert Frankfurts artenreichste grüne Oase natürlich auch die vielgerühmte Königin der Blumen. Im Unterschied aber zu anderen Rosarien der Region (KüBLER 2011) stehen die stacheligen Schönen hier gleichsam stets in stiller Konkurrenz mit Tausenden von anderen Gewächsen, vor allem den namengebenden Palmen, den Kamelien, Orchideen und Bromelien, um nur einige der wertvollen Pflanzen-Sammlungen des 1868 gegründeten Gartens zu nennen. Im Unterschied zu seinen anderen Themengärten wie Steinund Rhododendrongarten, Bambus- und Stauden-Anlage, befindet sich dieser "Rosenhag" ganz prominent im Zentrum des Palmengartens, gleich gegenüber dem Eingangsschauhaus an der Siesmayerstraße. Zwischen 2007 und 2008 wurde das in klassischer Manier streng geometrisch gestaltete Rosenparterre gründlich saniert. Bis es 1986 an dieser Stelle seinen vermutlich endgültigen Platz gefunden hatte, war freilich bereits über ein Jahrhundert Gartengeschichte vergangen.

In den Anfangsjahren kultivierte man zunächst nur Topfrosen im Palmengarten, immerhin in stattlicher Menge: 2000 Rosenstöcke in Kübeln sollen bereits 1869 mit dem reichen Pflanzen-Schatz aus den damals weithin be- 


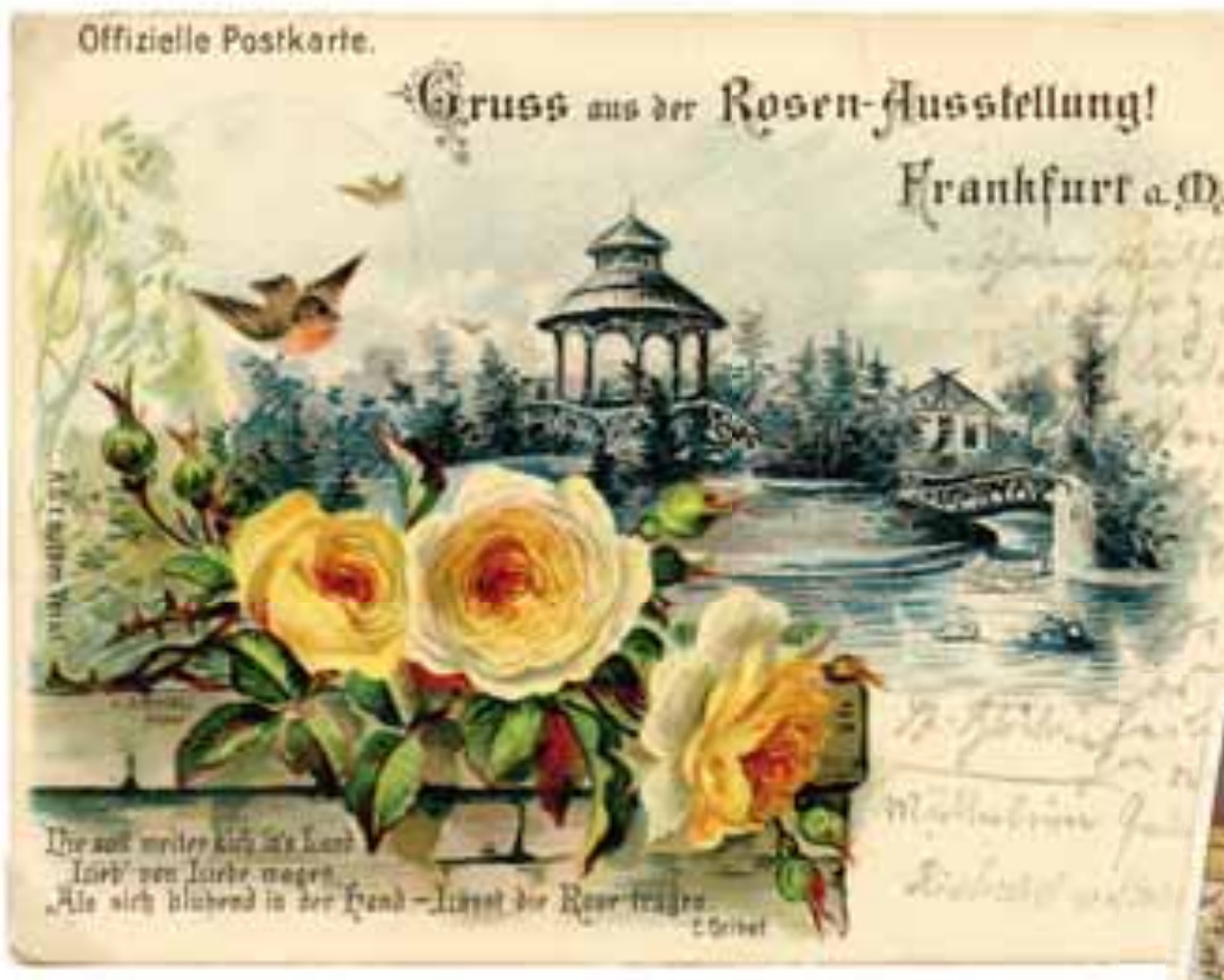

kannten Biebricher Wintergärten des Herzogs Adolph von Nassau von Wiesbaden nach Frankfurt gekommen sein (SCHOSER 1995). Im Palmengarten, der Monate zuvor von dem Bockenheimer Kunst- und Handelsgärtner HeinRICH SiesMAYER in einer legendären Bürgeraktion mithilfe einer „Actiengesellschaft“ gegründet worden war, fanden die herzoglichen PflanzenSammlungen alsbald ein prächtiges Obdach.

\section{Rosen im historischen Festsaal und königlicher Besuch}

Die Rosen wurden in den gleichfalls aus Biebrich stammenden Gewächshäusern vorgetrieben, um, in voller Blüte geschnitten, schon 1870 die erste Blumenschau im Palmengarten zu bereichern. Auch dienten die Rosen - wie

Abb. 1 (oben): Allgemeine Blumen- und Rosenausstellung 1897 in Frankfurt am Main. Offizielle Postkarte. Gebraucht, am 23.8.1898 von Frankfurt nach Braunschweig verschickt, angekommen am 24.8.1998.

Abb. 2 (unten): Allgemeine Blumen- und Rosenausstellung 1897 in Frankfurt am Main. Offizielle Postkarte. Karte ungebraucht. zuvor schon in der Residenz des Herzogs - als romantisches Dekor bei legendären Feierlichkeiten. Historische Berichte und Bilder (z. B. ScHoser 1981) überliefern, wie für Bälle, Kostümfeste und andere Lustbarkeiten die Säulen im großen Festsaal mit in Tannengrün gesteckten Rosen so dicht umrankt wurden, dass die Gäste in einer immensen, blütenübersäten Pergola zu tanzen meinten. Nach der aufwendigen Sanierung des seit 2006 geschlossenen Gesellschaftshauses, die in einigen Monaten ihren Abschluss findet, wird auch der restaurierte historische Festsaal den Besuchern wieder in voller Pracht zugänglich sein.

Zur offiziellen Eröffnung des Palmengartens im Frühjahr 1871 war sogar Kronprinz FriedRICH von Preußen mit seiner Gemahlin, der englischen Prinzessin Victoria, aus Berlin nach Frankfurt gereist (Schoser 1995). Beide Hoheiten haben die Rosen und anderen Pflanzen-Arrangements bewundert. 


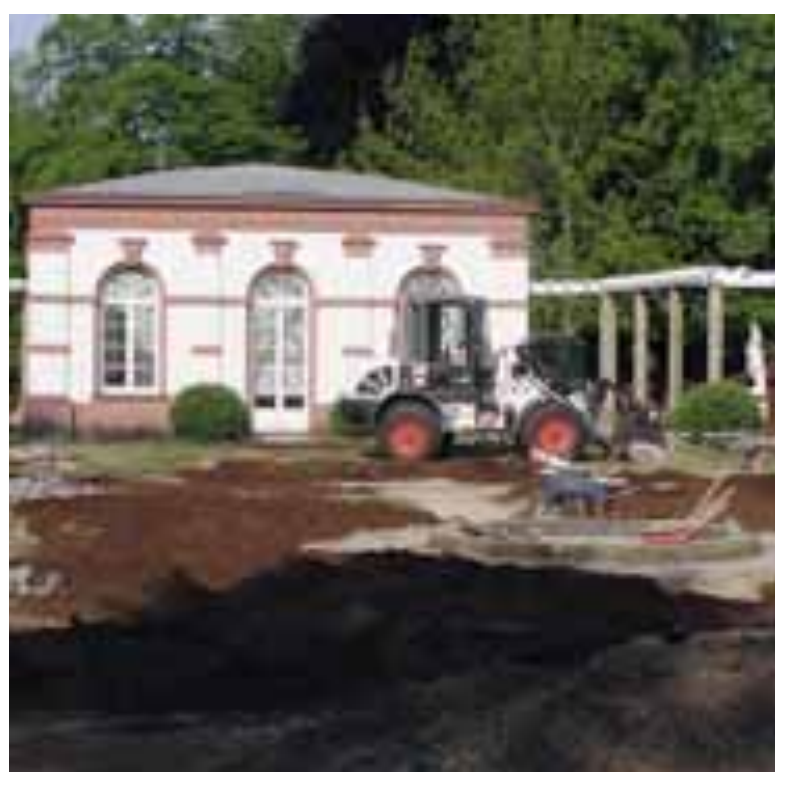

\section{Conrad Peter Strassheims Rosen für die Musterausstellung 1871}

Eine allererste Anlage nur für Rosen entstand im Palmengarten erst zur „Patent- und Musterausstellung" von 1881. Veranstaltet wurde diese Schau auf dem Gelände vor Haus Leonhardsbrunn, das damals noch nicht in den Palmengarten integriert war. Initiator und Gestalter dieses offenbar nur kurze Zeit überdauernden Rosengartens war der Bauunternehmer, Rosenliebhaber und -züchter Conrad Peter StrassHEIM. Er soll diesen Rosengarten sogar mit Pflanzen aus seinem eigenen Garten im damals noch ländlich geprägten Sachsenhausen bestückt haben (SCHOSER 1995).

Als Strassheim 1883 mit Gleichgesinnten den Verein Deutscher Rosenfreunde gründete, wurde er dessen erster Geschäftsführer. In dieser Funktion übernahm er auch die Redaktion der 1886 begründeten „Rosen-Zeitung“, die über Jahrzehnte hinweg das Kompendium schlechthin für Spezialisten, Sammler und Liebhaber der Königin der Blumen war, bis es von einem "Rosen-Bogen“ abgelöst wurde. Nach einer kurzzeitigen Wiederauflage der "Rosen-Zeitung" zwischen 1991 und 1993 wurde das Periodikum abermals in „Rosen-Bogen“ umbenannt. Herausgegeben und an die Mitglieder verteilt wird es nach wie vor von der unlängst in "Gesellschaft Deutscher Rosenfreunde" umbenannten Vereinigung in Baden-Baden.

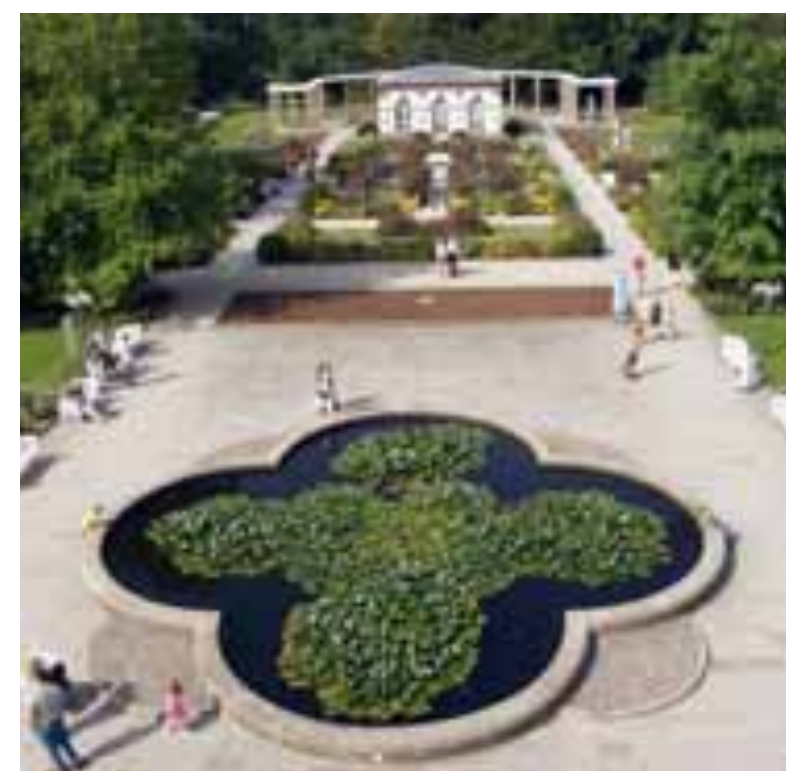

\section{SiesmaYers Nachfolger richtet den ersten Rosengarten ein}

Erst August Siebert legte den ersten dauerhaften Rosengarten an, nachdem der Palmengarten um ein stattliches Terrain erweitert worden war. Siebert hatte 1886 als nun hauptamtlich angestellter Gartendirektor den bis dato stets ehrenamtlich, also ohne Bezahlung den Park leitenden Gartengründer Heinrich SiesmayER, abgelöst. Schon bald nach seinem Amtsantritt konzipierte der neue Direktor den Rosengarten als weitere Attraktion in Form einer barock gestalteten Ellipse mit idyllischen Lauben, lauschigen Pergolen und rosenumrankten Sitznischen. Seinen Platz hatte dieser Rosenhag an der Schnittstelle zum 1885 angelegten Neugarten (Schoser 1995), etwa dort, wo heute das Tropicarium steht.

1893, zur 25-Jahr-Feier des Palmengartens, soll das stachelige Sortiment des Rosengartens 500 Varietäten umfasst haben (Schoser 1995).

Abb. 3 (oben links): Neugestaltung des Rosengartens. Die alten Pflanzen wurden abgeräumt, der Boden wurde ausgetauscht.

Abb. 4 (oben rechts): Blick vom historischen Eingangsschauhaus auf den Rosengarten mit Sommerflor als Übergangsbepflanzung, August 2007.

Abb. 5 (Seite 27): Der erneuerte Rosengarten, Sommer 2009. 
Siebert selbst beschreibt die Anlage 1895, also nur zwei Jahre später, in dem von ihm verfassten, vermutlich ersten Führer durch den Palmengarten wie folgt: „Das ganze Rosarium umfasst an Rosen aller Kategorien etwa 10000 Stück. Außer den zwei kleinen Rosenparterres ist die ganze Pflanzung irregulär gehalten, um den landschaftlichen Charakter durchgehend zu wahren; hoch- und halbstämmige wechseln mit Trauerrosen und niedrig veredelten $a b$. Von letzteren sind allgemein bevorzugte Sorten in größeren Trupps beisammen angepflanzt worden, um dem Auge in dem gemischten Blumenbild auch Ruhepunkte durch einzelne Hauptfarben zu gönnen und die Wirkung mächtiger zu gestalten. In Folge der häufig an uns gestellten Anfragen schien es von Wichtigkeit zu sein, dass hier angepflanzte Rosensortiment namentlich aufzuführen, und verweisen wir auf die Liste am Schlusse des Buches." (Siebert 1895).

Tatsächlich findet sich dort ein komplettes „Verzeichnis der in Töpfen und im Freien kultivierten Rosen“: rund 600 Sorten, in Gruppen eingeteilt und mit Namen und jeweiliger Blütenfarbe versehen, von einer lachsfarbigen 'Abbé Thomasson' über eine 'Kronprinzessin Victoria', die „milchweiss mit schwefelgelb“ blühte, bis hin zu einer 'Wellington', deren Flor SiEBERT als „leuchtend carminpurpur" beschreibt. Nach der Jahrhundertwende nennt Siebert in seinem „Führer durch den Palmengarten“ nur summarisch „eine Sammlung von etwa 3500 Rosen aller Kategorien: Hoch- und halbstämmige Rosen wechseln mit Trauerrosen und niedrig veredelten ab, Pavillons und Lauben, mit Schlingrosen berankt, verschönern das Bild.“ (Siebert o.J.).

Als Siebert, der sich noch „Königlicher Gartenbaudirektor" nennen durfte, 1923 starb, übernahm der bisherige Garteninspektor Отто Krauss die Direktion des Palmengartens. Unter seiner Leitung wurde der Rosengarten 1926 verlegt, und zwar an den Rand jener platzgreifenden Tennisplätze, die dort seit Beginn des Jahrhunderts ihr Domizil hatten und erst in den 1980er Jahren aus dem Garten verbannt

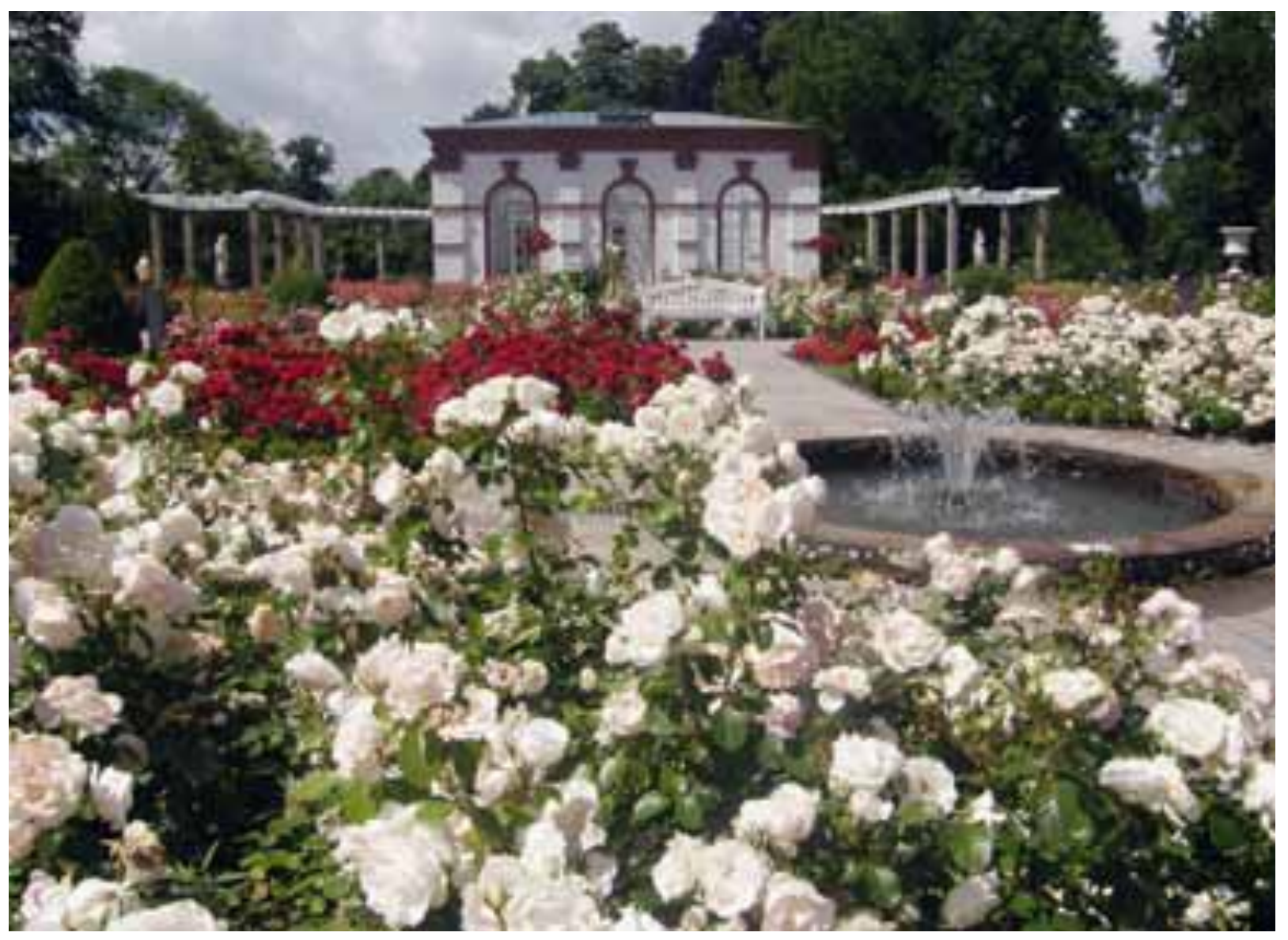


werden konnten. Dort wuchsen die Rosen nun auf einer 0,4 Hektar großen Fläche zwischen kubenförmig beschnittenen Hecken (siehe auch RöDEL 2001).

Wie bei allen Rosengärten war auch in dieser Anlage irgendwann der Boden ausgelaugt, so dass sie 1962 noch einmal frisches Substrat erhielt und neu gestaltet wurde (Schoser 1995). Der Nachteil: eine Kaltluft-Schneise aus dem Taunus, die noch nicht wie heute durch vielerlei Bebauung jenseits der Gartengrenzen und dank des heute hoch in den Himmel ragenden alten Baumbestands gebremst wurde, sorgte alle Jahre wieder für eine verspätete Rosenblüte. Die abermalige Verlegung der Rosen wurde deshalb schon damals für spätere Zeiten ins Auge gefasst.

\section{Ein neuer Rosengarten und der 1. Europäische Rosen-Kongress}

Seinen jetzigen und vermutlich endgültigen Platz erhielt der Garten der wehrhaften Blütenwunder während der 1980er Jahre im Rahmen der gigantischen Umbauphase und sogenannten „Inneren Erweiterung“ des Palmengartens unter dem damaligen Direktor Gustav SchoSER. Wiederum formal gestaltet, erstreckt sich seither ein romantisch anmutendes, luftiges Rosenparterre zwischen Palmenhaus-Galerien und Oktogon-Brunnen. Das Konzept für die Neu-Anlage stammte von Josef RafF, dem damaligen Gartendirektor der Insel Mainau (Schoser 1993).

Mitten aus den Beeten erhebt sich als zierlicher Pavillon das Haus Rosenbrunn, in dessen malerischem Ambiente sich inzwischen Hochzeitspaare standesamtlich trauen lassen können. Es ist kein Überbleibsel aus alten Tagen, sondern wurde seinerzeit neu errichtet aus alten Mauer- und Fassadensteinen des historischen Betriebsgebäudes von 1882 (SCHOSER 1995), das bis dahin den prominenten Platz besetzt hatte.

Zur Eröffnung der neuen Rosen-Anlage im Juni 1988 konnte Schoser als tatkräftiger Bauherr und international angesehener Botschafter von vielerlei Pflanzengruppen sogar den 1 . Europäischen Rosenkongress nach Frankfurt ho- len. So strömten damals Rosenzüchter, Botaniker und Gärtner mit Abertausenden von Rosenliebhabern im Gefolge zur neuen Attraktion des Palmengartens.

\section{Das Rosen- und Lichterfest}

Auch das Feiern mit Rosen hat eine lange Tradition im Palmengarten, wie oben bereits erwähnt wurde: Zu Beginn der 1930er Jahre ließ der damalige Direktor Max Bromme die Rosenschauen der Anfangszeit wieder aufleben und erweiterte sie zu floristischen Musterschauen, die alsbald großen Anklang bei den Besuchern fanden. In seiner Funktion als städtischer Gartendirektor hatte Bromme 1931, als der Palmengarten in städtischen Besitz überging, auch dessen Leitung übernommen und als Nachfolgerin der bisherigen „Actiengesellschaft" die "Gesellschaft der Freunde des Palmengartens" ins Leben gerufen. Seine Pläne für einen Rosenbewertungs-Garten, den er 1933 bei der 50-Jahr-Feier des Vereins Deutscher Rosenfreunde im Palmengarten präsentiert hatte, konnte er indes nicht realisieren: Nach ersten Rosenpflanzungen musste das Projekt mit Ausbruch des Zweiten Weltkriegs eingestellt werden (SChoser 1995).

Das 1932 begründete Rosen- und Lichterfest, das mit Ausnahme der Kriegs- und Nachkriegsjahre bis heute gefeiert wird, geht ebenfalls auf Bromme zurück. Seither bildet diese Veranstaltung einen in jedem Juni wiederkehrenden Höhepunkt im Palmengarten-Jahr. Im Zentrum des umfangreichen dreitägigen Kultur-Programms zum Thema Rosen steht ein duftendes, vielfarbiges Szenario aus abertausenden Rosenblüten. Mit mancherlei Ausnahme von der Regel wird diese Schau in der Galerie am Palmenhaus gezeigt. Längst weit über die Grenzen Frankfurts hinaus bekannt und beliebt, lockt das Fest alljährlich zigtausend Garten- und Rosenfreunde ins Westend, vor allem zum fulminanten nächtlichen Feuerwerk und der Illumination des Gartens: Von den Besuchern im gesamten Park verteilt, erhellen dann unzählige flackernde Teelichter die meist schon laue Frühsommernacht (vgl. auch TAudTeREPP 2008). 

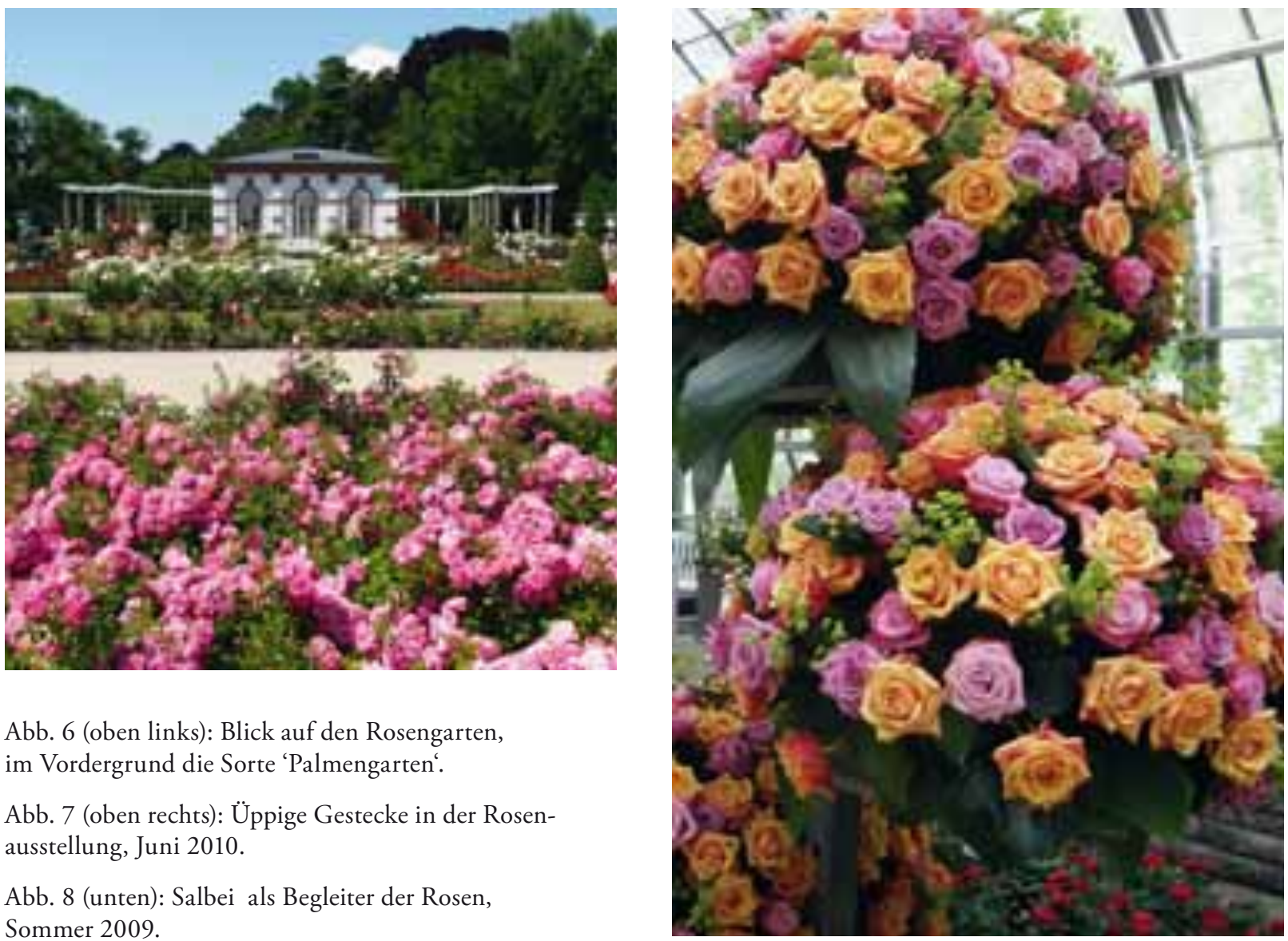

Abb. 6 (oben links): Blick auf den Rosengarten, im Vordergrund die Sorte 'Palmengarten'.

Abb. 7 (oben rechts): Üppige Gestecke in der Rosenausstellung, Juni 2010.

Abb. 8 (unten): Salbei als Begleiter der Rosen, Sommer 2009.

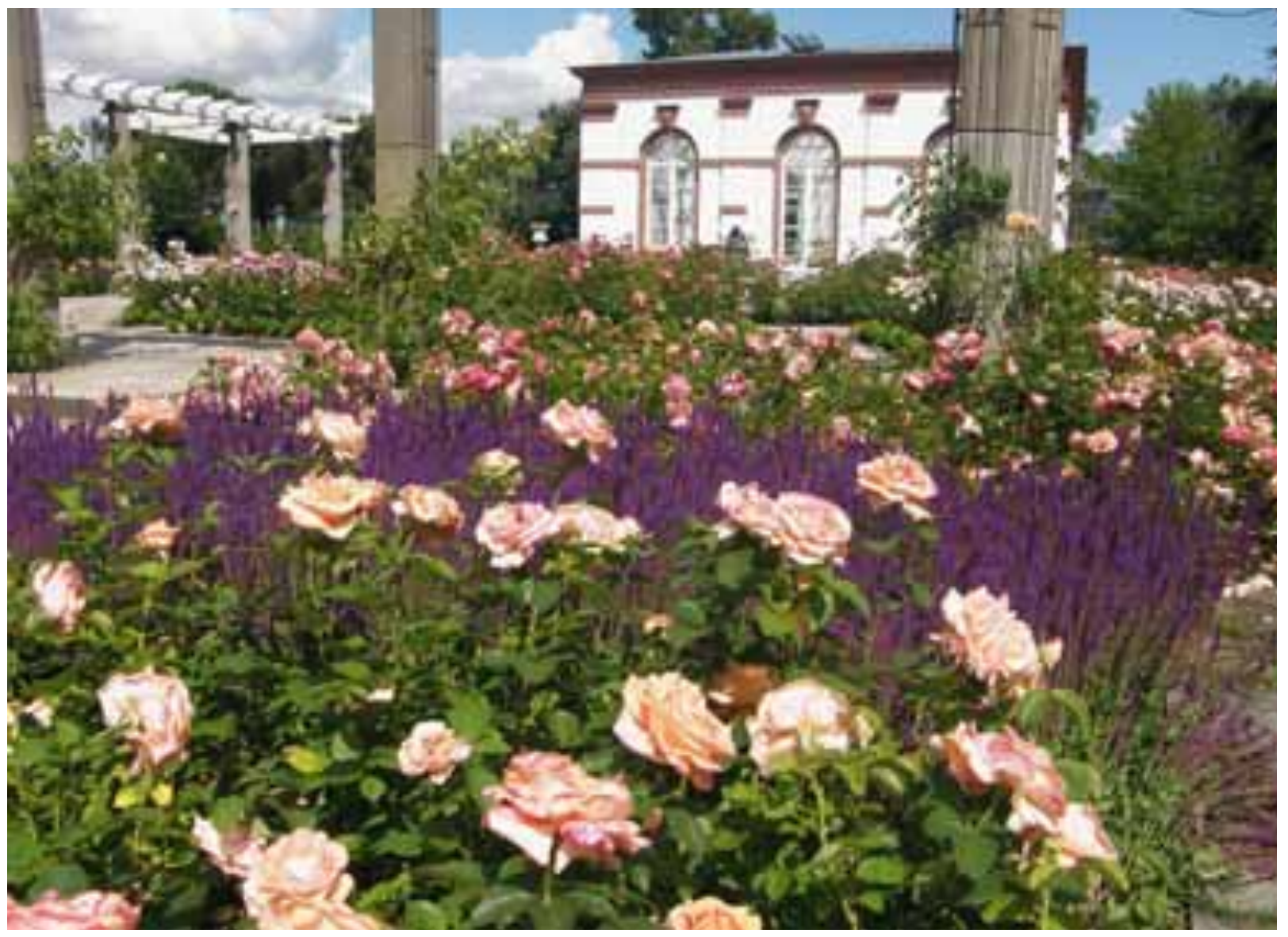




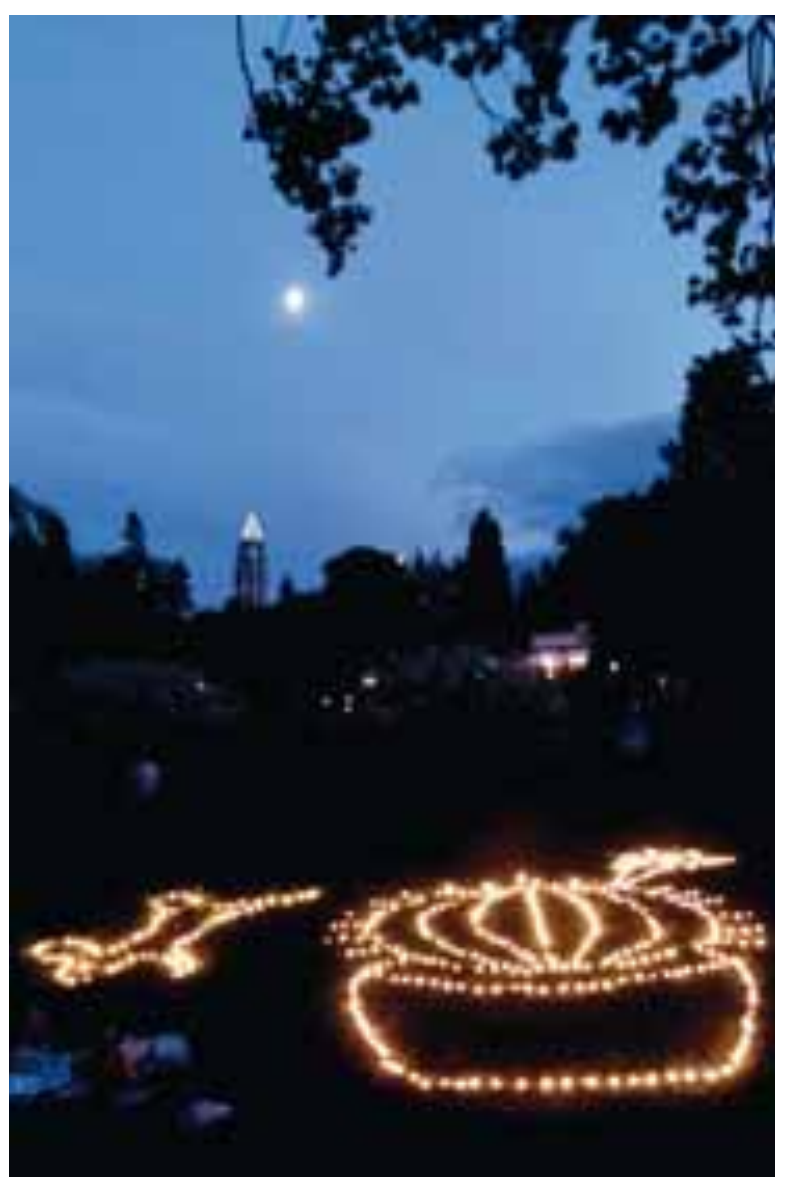

Zum Rosenfest im Jahr 2010 stiftete die Stadt Lyon ein weiteres, ganzjährig zu bestaunendes optisches Highlight. Der Designer JACQUes Fournier aus Frankfurts ältester Partnerstadt in Frankreich, die alljährlich im Dezember ihr traditionsreiches Lichterfest („Fête de Lumières“) feiert, entwickelte für den 25 Jahre alten Oktogonbrunnen ein raffiniertes künstlerisches Lichtkonzept mit sechs Dutzend bunten LED-Spots (KLIMEK \& Jenny 2011).

\section{Sanierung und Neugestaltung mit Steinfurther Rosen}

Die unlängst wieder notwendige Sanierung des ermüdeten Erdreichs nutzte der Palmengarten unter seinem derzeitigen Direktor Matthias JeNNY zwischen 2007 und 2008 für eine behutsame Neuanlage des Rosenparterres. So wurden die Wege mit Natursteinplatten erneuert, eine stimmungsvolle Beleuchtungsanlage installiert und alle Beete mit niedrig gehaltenem Buchsbaum eingefasst. Etwa 50 verschiedene Sorten finden sich unter den durchweg

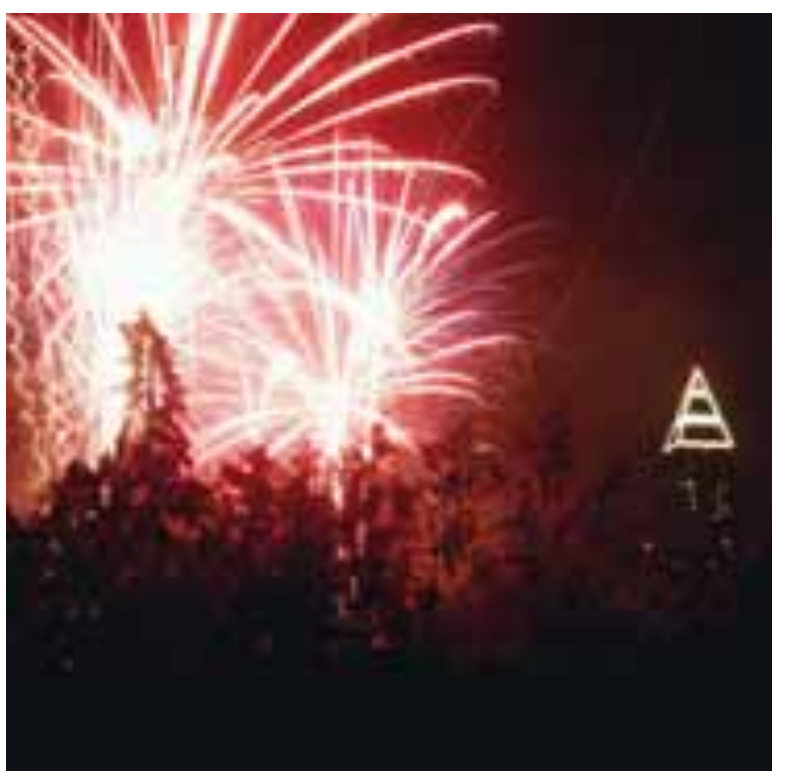

neu gepflanzten Rosenstöcken. Die Mehrzahl wurde dem Palmengarten von der Steinfurther Rosen-Union gestiftet. Zum harmonischen Gesamtbild der Anlage trägt nun eine subtile Farbregie bei.

Ein breites Band der rosa blühenden 'Palmengarten Frankfurt' schwört den Besucher schon jenseits der Haupt-Allee auf diese kleine Welt der Königin der Blumen ein. In kräftigem Gelb leuchtende Strauchrosen unterschiedlicher Sorten flankieren das Parterre. Von der prachtvollen 'Red Leonardo da Vinci' gefasst, hat man in die zentralen Beete rot und weiß blühende Sorten wie 'Happy Wanderer' oder 'Princess of Wales' gepflanzt - als Hommage an die Farben des Frankfurter Stadtwappens.

Hochstämmchen wie 'Aspirin' oder 'Roter Korsar' sowie hie und da untergemischter dunkelblauer Salbei (Salvia nemorosa) sowie ganz klassisch zu Pyramiden oder kugelförmig geschnittene Buchsbäume verleihen dem Ensem-

Abb. 9 (oben links): „Teelichtkaktus“ während des Rosen- und Lichterfestes 2011.

Abb. 10 (oben rechts): Das traditionelle Feuerwerk beim Rosen- und Lichterfest.

Abb. 11 (Seite 31 oben): Raureifstimmung im Rosengarten.

Abb. 12 (Seite 31 unten): Vom Schnee überraschte Rosen, 24. November 2008. 
ble eine einladend-heitere Atmosphäre. Bequeme weißlackierte Holzbänke, ein zentrales Wasserbecken mit kleiner Fontäne und schmucke historische Amphoren runden das harmonische Gesamtbild ab. Gleiches gilt für die in Rosé und Magenta gehaltenen Beete rund um Haus Rosenbrunn. Die Sorte 'Dolly' behält ihren Flor oft sogar bis weit in den Winter hinein.

Hinter dem auch für Ausstellungen genutzten Pavillon gibt es Beetrosen in Gelb, Orange und Apricot. Sorten von Kletterrosen wie 'Kir Royal' oder 'New Dawn', die an den Säulen der Pergola stehen, zeigen verschiedene Farbtöne. Die drei „Frühling“, „Sommer“ und „Herbst“ symbolisierenden Marmorfiguren verdankt der Palmengarten wie die oben erwähnten Amphoren einer frühen Schenkung der Familie RoтнSCHILD (SCHOSER 1995).

Ein neuer Duftgarten wurde ebenfalls bei den jüngsten Sanierungsmaßnahmen angelegt. Er befindet sich vor der Palmenhaus-Galerie und umfasst verschiedene Rosensorten, die mit Aromapflanzen wie Lavendel und Salbei sowie mit Sedum, Schafgarbe oder Frauenmantel kombiniert sind. Leuchtend gelbe Narzissen bilden hier im zeitigen Frühjahr duftende Vorboten der kommenden Blütenvielfalt.

Auch wenn ihnen je nach Saison die Kamelien und Orchideen, die Azaleen und Rhododendren, die Frühlingsgeophyten oder der Sommer- und Herbstflor die Schau zu stehlen scheinen, sind die Rosen im Palmengarten doch bis heute ebenso präsent wie prominent, vor allem in den Sommermonaten, als blütenreiches Empfangskomitee für alle Besucher, die von der Siesmayerstraße aus den Garten betreten.

\section{Literatur}

KlimeK, S. \& Jenny, M. 2011: Palmengarten 2010. - Palmengarten 75: 1-11.

KüBLER, I. (Hrsg.) 2011: Stachlige Schönheiten. Die Rosengärten in Frankfurt-Rhein-Main. - Frankfurt. RöDel, V. 2001: Aus der Geschichte des Palmengartens. - Palmengarten 65: 118-131.

Schoser, G. 1981: Ein Tor zur Welt der Pflanzen. Der Palmengarten in Frankfurt am Main. - Frankfurt. Schoser, G. 1993: 125 Jahre Palmengarten Frankfurt am Main (Informationsheft zur gleichnamigen Ausstellung). - Frankfurt.
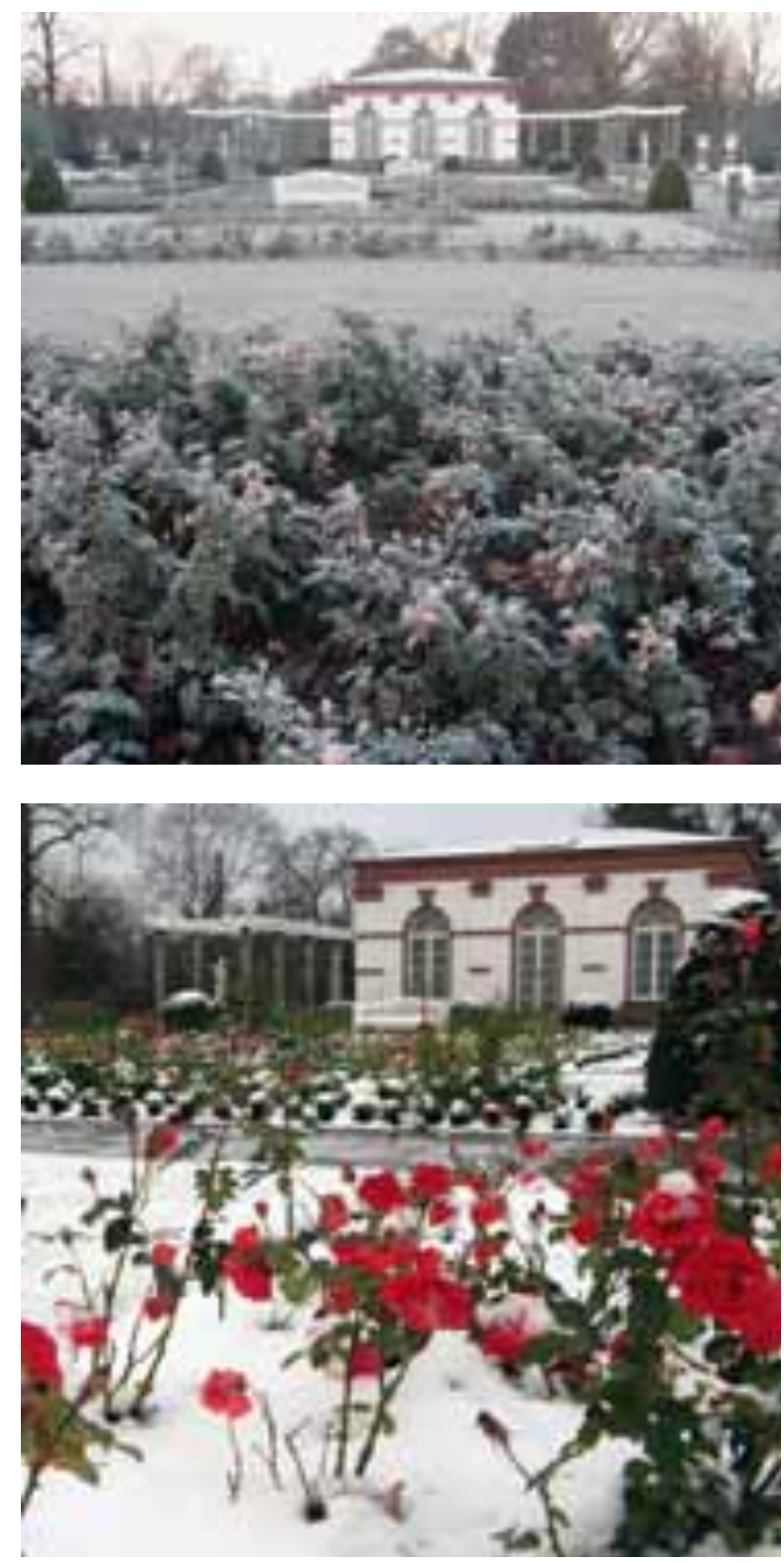

Schoser, G. 1995: Eine Welt der Pflanzen. Palmengarten Frankfurt. A World of plants. - Frankfurt, Baden-Baden. Siebert, A. 1895: Der Palmengarten zu Frankfurt a. M. Geschichte und Beschreibung. - Berlin. (Eine digitalisierte Fassung des Bandes findet sich im Internet:

http://www.archive.org/stream/

derpalmengarten00siebgoog\#page/n4/mode/2up)

Siebert, A. o. J.: Führer durch den Palmengarten in Frankfurt am Main. - Frankfurt. (Dieser Führer erschien in verschiedenen, auch unterschiedlich gestalteten Auflagen und Ausgaben ab ca. 1903, wie sich aus je aktuellen inhaltlichen Angaben schließen lässt.)

Taudte-Repp, B. 2008: Der Palmengarten. Ein Führer durch Frankfurts Grüne Oase. 2. Aufl.- Frankfurt. 